\title{
Neuro-Cutaneous Syndrome (NCS) (Morgellons) (Delusional Parasitosis) A Dental Toxicity Disorder
}

\author{
Omar M Amin* \\ Parasitology Center, Scottsdale, Arizona \\ *Corresponding Author: Omar M Amin, Parasitology Center, Scottsdale, Arizona.
}

Received: July 12, 2019; Published: October 28, 2019

DOI: 10.31080/ASMI.2019.02.0411

\section{Background}

At the Parasitology Center, Inc. (PCI), we have been researching Neurocutaneous Syndrome (NCS) in over 1000 cases since 1996. Our early reports on this syndrome included the description of a case with many facial opportunistic infections from Oklahoma [1] and the first naming and evaluation of the syndrome from 3 more cases, with a special reference to fibers and springtails (Collembola) [2]. By 2003, we were able to provide a comprehensive evaluation of NCS and establish the link to dental toxins as the causative agents. Amin [3] clarified the nature of action of dental liners (bases) in the causation of NCS neurological and dermatological symptoms and provided the history of 3 NCS patients who have recovered following rehabilitation thus establishing a cause-effect relationship. Various versions of this landmark publication were subsequently published elsewhere [4-16].

The above contributions, among others, were researched and published, and patients were successfully helped long before we discovered a similar clinical entity called Morgellons. The only difference is that we, at PCI, have done the research, established a causal relationship with dental toxins, developed a protocol, and successfully helped patients.

Research on the relationship between NCS and dental toxicities was first published in Explore 12: 21-25, 2003.

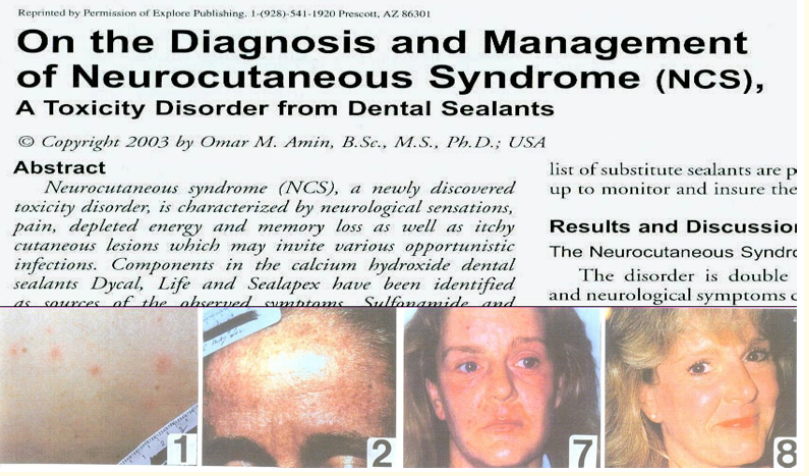

Figure 1

Original research was also published in the: California Dent. Assoc. J. 2004, Vol. Vol. 32 (9): 657-663.

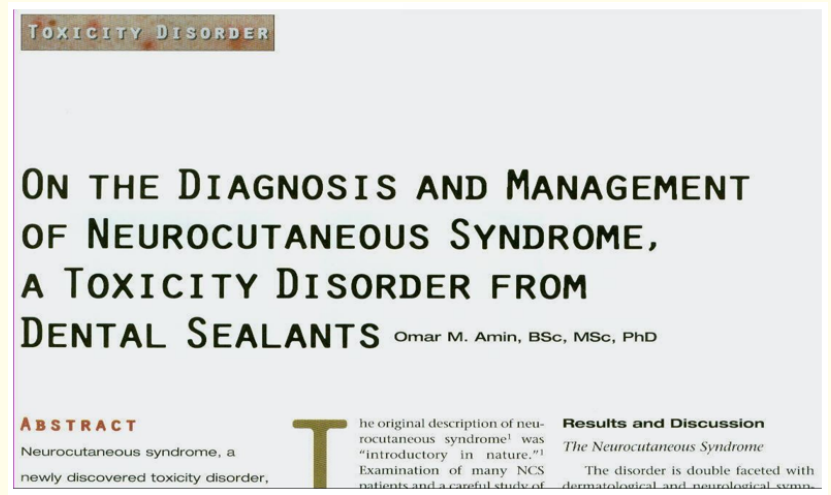

Figure 2 
Neurocutaneous Syndrome (NCS), a newly discovered dental toxicity disorder, is characterized by neurological (pin-prick and movement) sensations (often confused with parasites and diagnosed as delusional parasitosis), pain, depleted energy, and memory loss, as well as itchy cutaneous lesions that may invite various opportunistic infections. Components in the calcium hydroxide Dycal, Life, and Sealapex materials, among others, have been identified as sources of the observed symptoms.

Toxicity issues and additional notes on zinc oxide, Fynal, IRM, and Sultan U/P sealants, among other sealants and toxic dental materials are also included.

Over 20 case histories and management protocols are also discussed.

\begin{tabular}{|c|c|c|c|}
\hline Material & Dycal- & Life* & Sealapex \\
\hline $\begin{array}{l}\text { Calcium } \\
\text { hydroxide }\end{array}$ & $51 \%(C)$ & $51 \%(B)$ & NG $(B) \cdots$ \\
\hline Zinc oxide & $9.23 \%(C)$ & $13.75 \%(B)$ & NG (B) \\
\hline Zinc stearate & $0.29 \%(C)$ & $0.25 \%(B)$ & \\
\hline Ethyitoluene sulfonamide & $39.48 \%(C)$ & $34 \%(B)$ & NG (B) \\
\hline Silicon dioxide & - & - & $N G(B)$ \\
\hline Titanium dioxide pigment & $\longrightarrow$ & $10.0 \%(\mathrm{C})$ & NG (C) \\
\hline Pigment & $0.1 \%(B)$ & $0.1 \%(C)$ & \\
\hline Calcium phosphate & $31.0 \%(B)$ & $\longrightarrow$ & 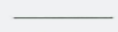 \\
\hline Barium sulphate & $\longrightarrow$ & $37.90 \%(C)$ & NG (C) \\
\hline Zinc oxide & $9.0 \%(B)$ & $\longrightarrow$ & - \\
\hline Methyl silicate & $\longrightarrow$ & $12.0 \%(\mathrm{C})$ & 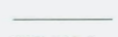 \\
\hline Silicon dioxide & $\longrightarrow$ & $\longrightarrow$ & NG (C) \\
\hline Calcium tungstate & $17.0 \%(B)$ & $\longrightarrow$ & - \\
\hline Butylene glycol disalicylate & $43 \%(B)$ & $\longrightarrow$ & $\longrightarrow$ \\
\hline $\begin{array}{l}\text { Polymethylene mythyl } \\
\text { salicylate }\end{array}$ & 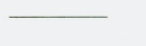 & $38.0 \%(C)$ & \\
\hline Isobutyl salicylate & - & $\longrightarrow$ & NG (C) \\
\hline
\end{tabular}

Figure 3

Toxicity of components in liners/bases

- $\quad$ The major calcium hydroxide liners (bases) causing NCS (Dycal, Life, Sealapex) include only ca. 50\% calcium hydroxide in the catalyst.

- Zinc oxide and ethyltoluene sulfonamide are most toxic.

- $\quad$ Toluene is a known potent nerve toxin associated with the neurological symptoms in NCS patients.

- The sulfonamide component brings about a sensitivity allergic toxic reaction ultimately presenting as vascular mucoid sores, compatible with classical sulfa toxicity symptoms, especially in sulfa sensitive patients.
- Titanium dioxide and Barium ions provoke strong foreign body and bio-incompatible reactions in live tissues.

Toxicity of sealapex (example \#1)

- $\quad$ Sealapex was shown to cause severe inflammatory infiltration and edema accompanied by subcutaneous tissue necrosis and progressive differentiation and reaction of monocytes, macrophages, and epithelial cells.

- The final phase of inflammation is characterized by an intense granulomatus reaction especially in epithelial cells causing various intensities of irritation.

- The cytotoxicity and neurotoxicity of Sealapex was well demonstrated in vivo (in various mammalian systems) and in vitro (in a variety of tissues).

Toxicity of dycal and life (example \# 2)

- Dycal and Life have been shown to cause hemorrhage and acute to consistent inflammatory cell necrosis, tissue loss, karyorrhexis, neurotoxicity, and formation of serous exodate.

- $\quad$ Both Life and Dycal have been frequently associated with classical NCS symptoms in many of our patients.

- Sealants not containing ethyltoluene sulfonamide but including zinc oxide and eugenol, e.g., Fynal, IRM, and Sul$\tan \mathrm{U} / \mathrm{P}$ were also associated with NCS cases.

Toxicity of components in liners/bases

DB. White female from California born 4-9-1965

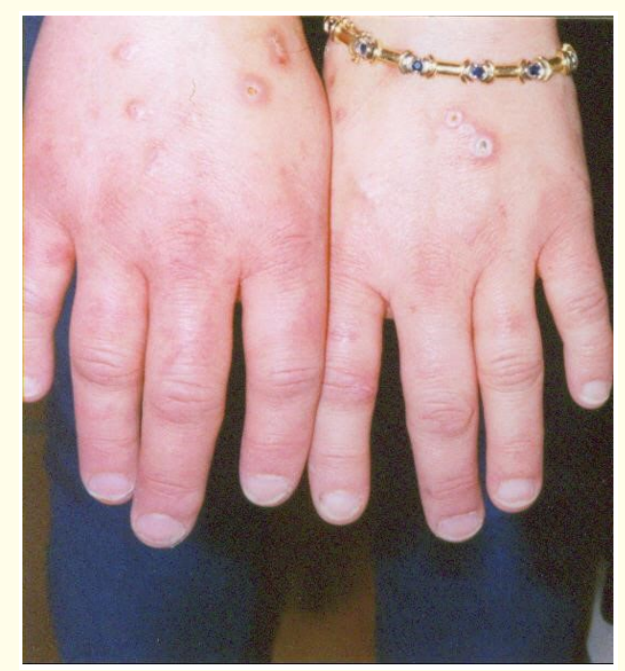

Figure 4 
- Had 10 amalgam restorations in 1982-83 using Life.

- $\quad$ Symptoms first noted in 2001-02: Sores, swelling, diffuse rash, black specks (fungal spores), pin prick and subcutaneous crawling sensations.

- Diagnosed with anxiety dis-order. Sores dismissed as not clinically genuine and patient declared normal.

- $\quad$ Diagnosed with NCS at PCI on 1212-02. Treatment in progress.

ME: White female from Sweden born on 5-26-51

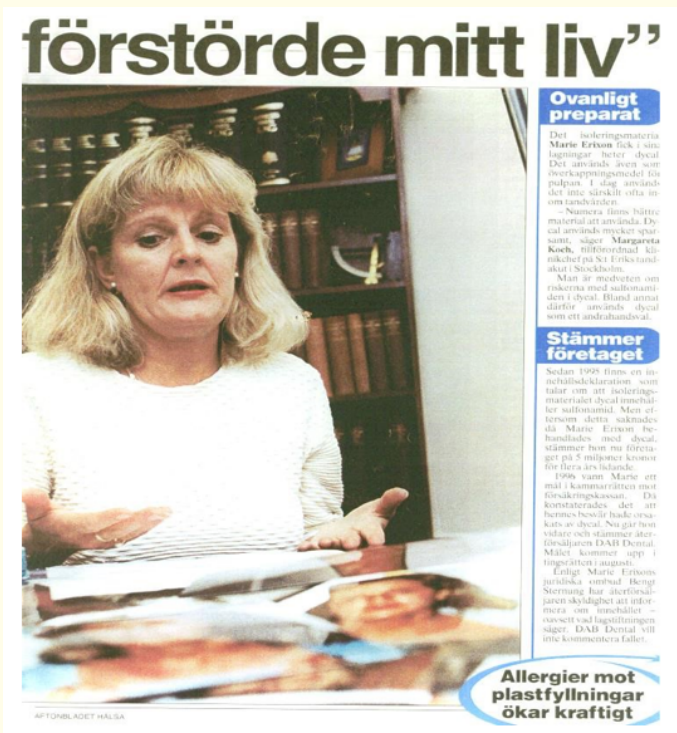

Figure 5

- A businesswoman from Sweden now turned health care practitioner, had Dycal in 20 teeth in 1985.

- $\quad$ She is allergic to sulfa.

- Dycal was removed in 1991 and 1992 and initially replaced with Harvard cement. Initial healing period was very painful.

ME (cont.): Had typical sulfa toxicity symptoms, oozing skin and nasal sores, memory loss, kidney pain, sensitivity to light and electricity, pin prick and crawling sensations, itching and breathing difficulties.
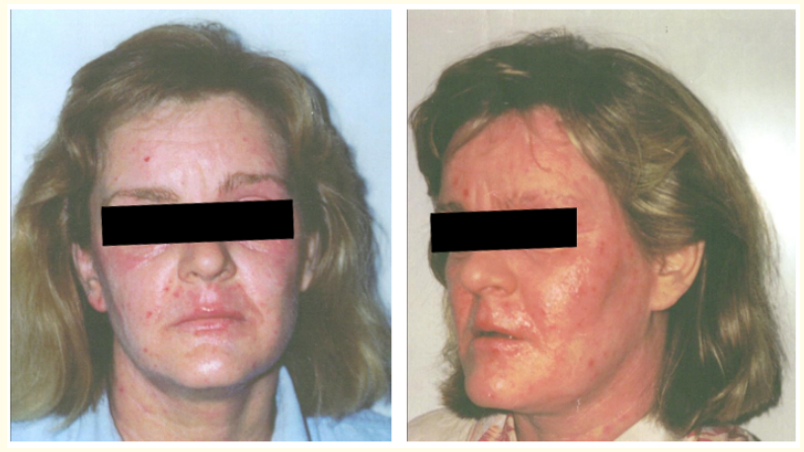

Figure 6

ME (cont.): Photosensitivity produced blotchy skin and enhanced the diffusion of sores accompanied by severe burning sensations in the face, throat and chest. Fatigue and headaches were relentless.

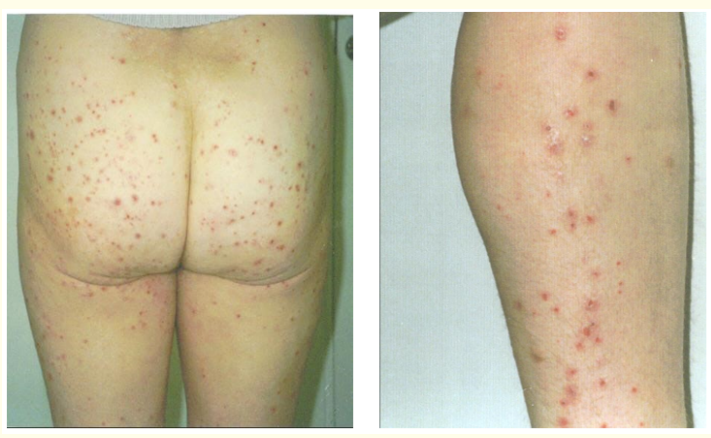

Figure 7

ME (cont.): Diagnosed with polyneuropathy, atopic dermatitis, toxic (Dycal-related) ulcerative dermatitis. Experienced severe musculoskeletal pain and bowel disturbances during initial stages of dental rehabilitation. Complete recovery in 1992 after one year of rehabilitation. Now: activist against Kerr Co., USA. 


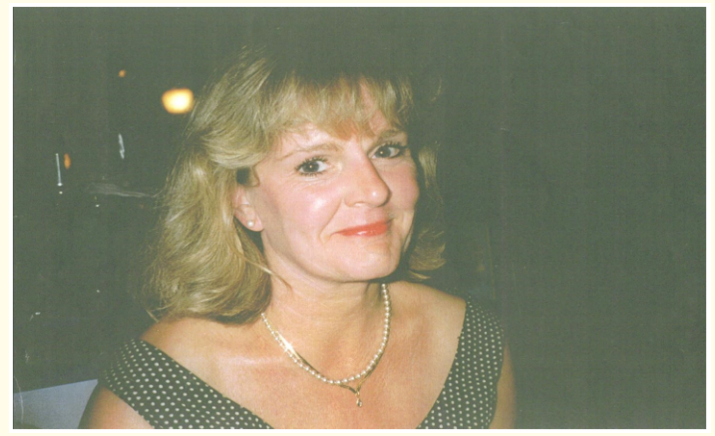

Figure 8

LG: White female from California born in 1957
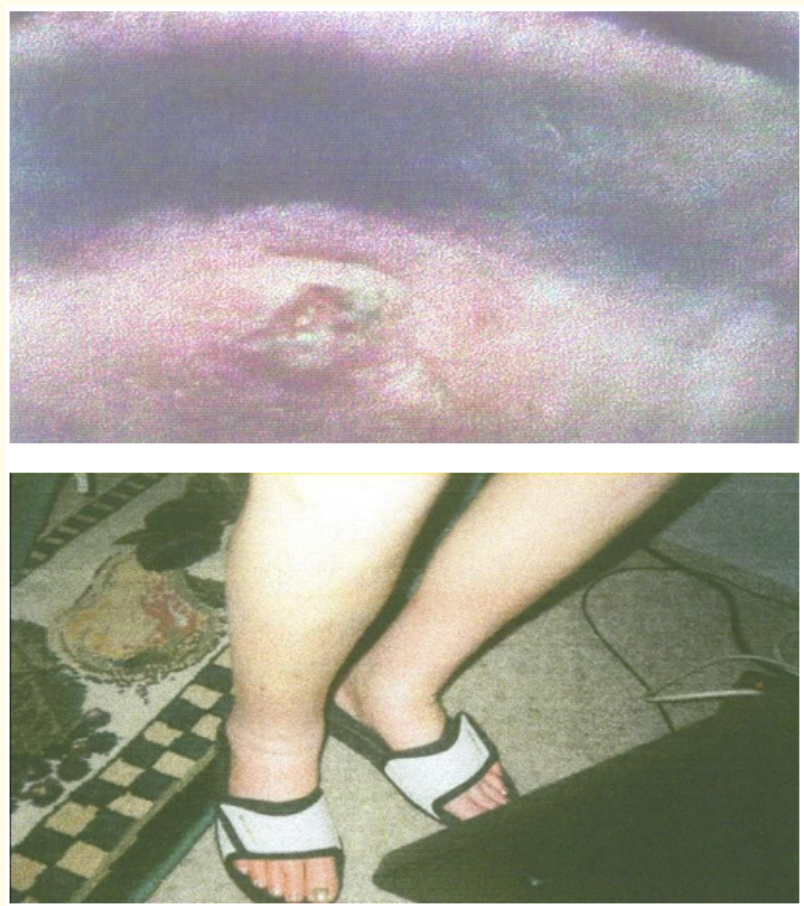

Figure 9

- $\quad$ Dycal in tooth \# 18 on 9-18-98. Allergic to sulfa. Symptoms started same day (shortest incubation period noted) with vomiting and headache.

- Other symptoms: Lesions, open sores with grayish secretions (top right) springtails and fibers, pin prick and movement sensations, high blood pressure, fatigue, heart palpitation and mitral valve prolapse, swelling (bottom right) and weight loss.
- Diagnosed with self-inflicted wounds, scabies (no mites demonstrated), stress, anxiety disorder, crazy (almost admitted to insane asylum).

- $\quad$ Diagnosed with NCS at PCI in Jan. 2002.

- $\quad$ Removed Dycal in April, 2002 and replaced with Starflow and Aria.

- All symptoms resolved by May, 2002 (shortest observed recovery) and remain resolved.

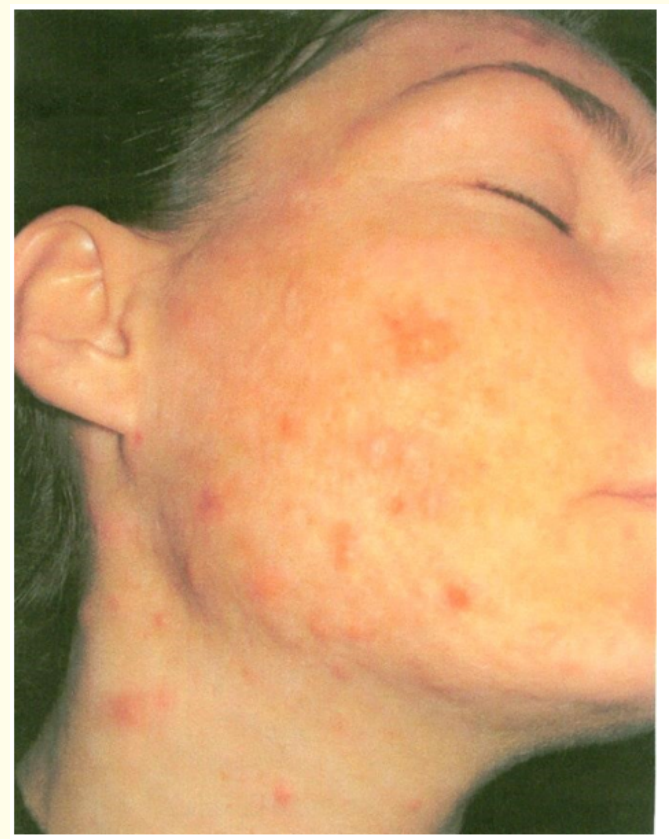

Figure 10

EJ: White female from Norway born on 5-22-1971

- 7 fillings using Dycal and Life with Clearfil SE primer and bond (2hydroxyethyl methacrylates, dimethacrylates) in 1979 (at age 7). Root canal in 1 tooth using Procosol in 1995. Highly reactive to Dycal and Life (bio-compatibility test).

- NCS symptoms first observed in 1989: Dental decay, sores, pin prick, crawling sensations, heart palpitations, memory, breathing and intestinal disturbances, swelling, fatigue, electromagnetic sensitivity, night fevers/sweats, insomnia, gray gum tissue and tongue, trauma. 


\section{EJ (cont.)}

- $\quad$ Diagnosed with delusional parasitosis, cancer, unspecific dermatitis, scabies (no skin samples taken, no mites/ parasites found). Treated with Dalacin, Tetracycline, Arco and Differ in without improvement.

- $\quad$ Diagnosed with NCS At PCI on 4-23-04. Completed dental rehabilitation using Durelon and Unicem cement in May and Aug, 2004.

- $\quad$ Symptoms have resolved. (Scan in April, 2004).

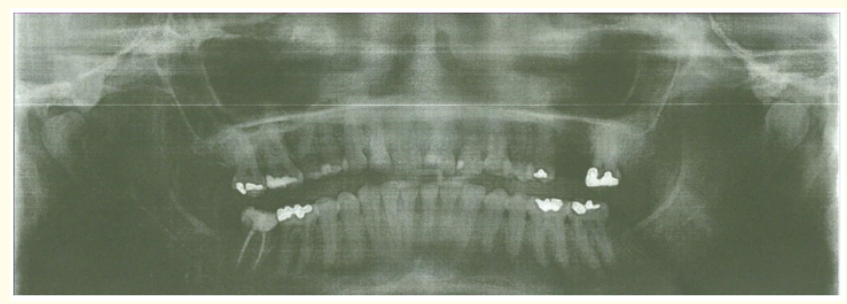

Figure 11

SK: White female from California born 9-17-1956

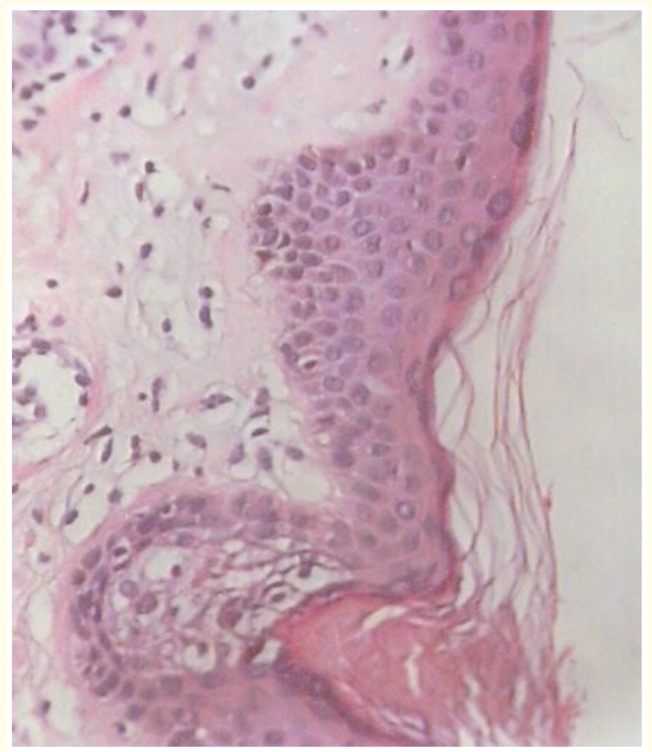

Figure 12

- $\quad$ Porcelain veneers cemented with Durelon (zinc oxide) in 1982.
- Symptoms: Pain, inflammation, widely diffused lesions, itching, shaking, fungal infections, recurrent reaction to sulfa drugs.

- $\quad$ Diagnosed with deep perivascular dermatitis. Treated with Vicodin, Augmentin, Tinidazole and cod liver oil.

- Diagnosed with NCS at PCI in Dec. 2002. Follow up information not available.

- A histopathological section in 1 of the more than 300 sores of SK showing hyperkeratosis-like perivascular dermatitis with eosinophils.

JM: White female from Arizona/Colorado born on 4-17-65

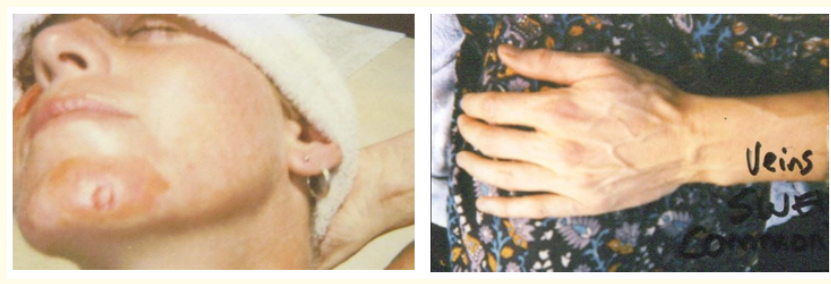

Figure 13

- 17 fillings; 16 with Dycal.

- $\quad$ First NCS symptoms in 1991

- Rash, ulcerations crawling and pin prick sensations, vomiting, joint and dental pain insomnia, swelling, skin peeling, coughing and elevated veins.

- Heavily medicated.

JM (cont.)
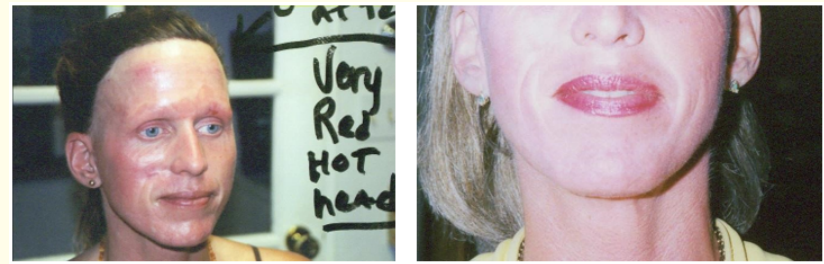

Figure 14

- JM also experienced red-hot face (left) and chest and night fevers.

- $\quad$ She was diagnosed as psychotic with delusional parasitosis. 
- Never taken seriously by medical professionals, family or friends.

- $\quad$ She was diagnosed with NCS at PCI in Sept. 2000.

- $\quad$ Dycal was removed in 2001. Followed with initial episodes of sickness, sweats and vomiting.

- $\quad$ Complete resolution of symptoms by mid-2002 (right).

MM: White female from California born 9-2-1950

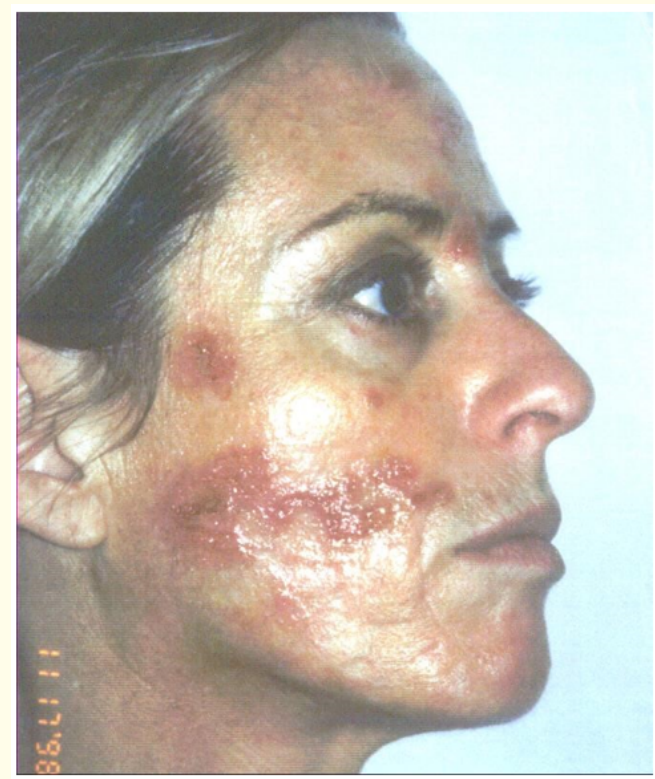

Figure 15

- $\quad$ Fynal in 6 teeth in 1981 and in one tooth in 1986; Life in 2 teeth in 1985 and 1988 (root canal, metal rods, impacted teeth, crowns.

- Highly reactive to Dycal and life (biocompatability test).

- Symptoms: mucoid lesions on face, pain, black specks (fungal spores), intense itching, excretions, ringing in ears, crawling sensations, poor circulation, compromised immunity. Photo (right) in 1982.

- $\quad$ Diagnosed with psychosis (1999), hyperkeratosis hemachromatosis, mercury poisoning, over-use of antibiotics (1998).

- $\quad$ Diagnosed with NCS at PCI in 1999.
MM (cont.) Dental rehabilitation in May and June, 2004

- May: Symptoms were "calm" at beginning of $1^{\text {st }}$ rehabilitation (left).

- June: Symptoms aggravated after $1^{\text {st }}$ rehab. at time of $2^{\text {nd }}$ rehab. (right).

- Currently: Marked improvement in all symptoms as of Aug. 2004.
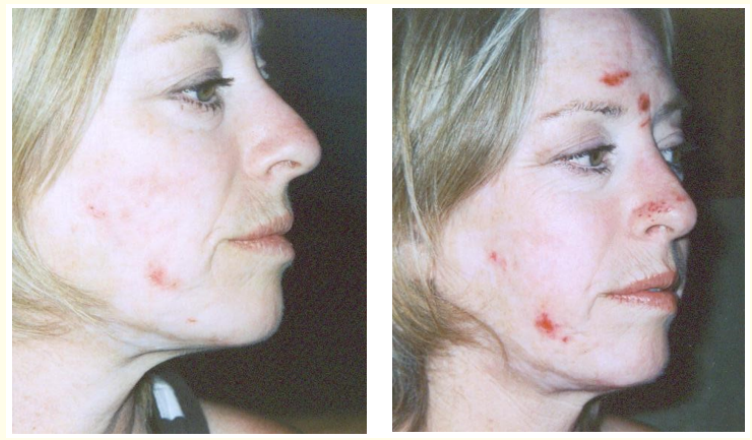

Figure 16

TR: White female from Nevada born on 10-8-64

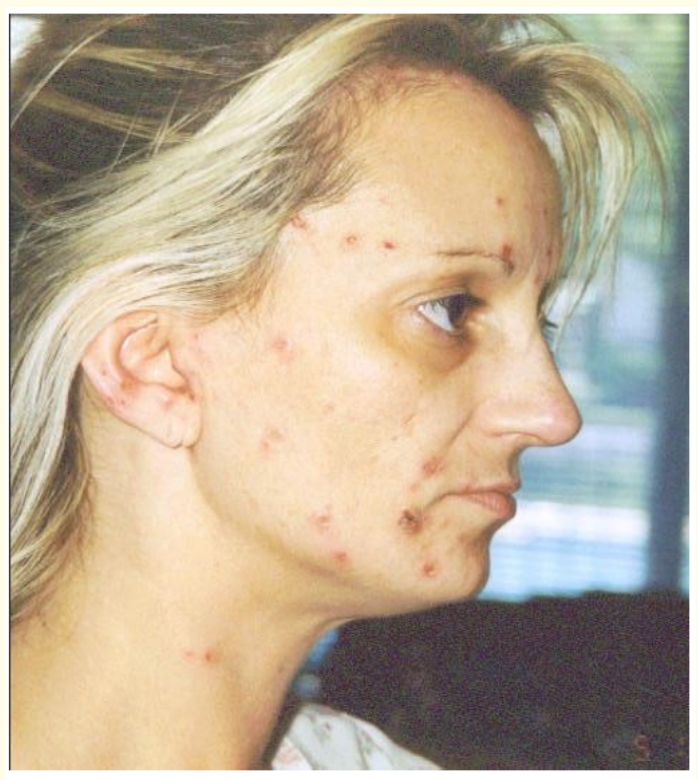

Figure 17 
- 5 molars using Dycal. Written records not available.

- Symptoms: diffused skin eruptions throughout entire body, severe itching, crawling sensations and fatigue.

- $\quad$ Diagnosed with delusional parasitosis and dermatitis.

- $\quad$ Diagnosed with NCS at PCI on 2-132003.

- $\quad$ Removed all compromised molars in Sept., 2003.

- $\quad$ All NCS symptoms resolved shortly after molar extraction.

TR (cont.): Diffuse skin eruptions in Feb., 2003 before dental restoration in Sept 2003.
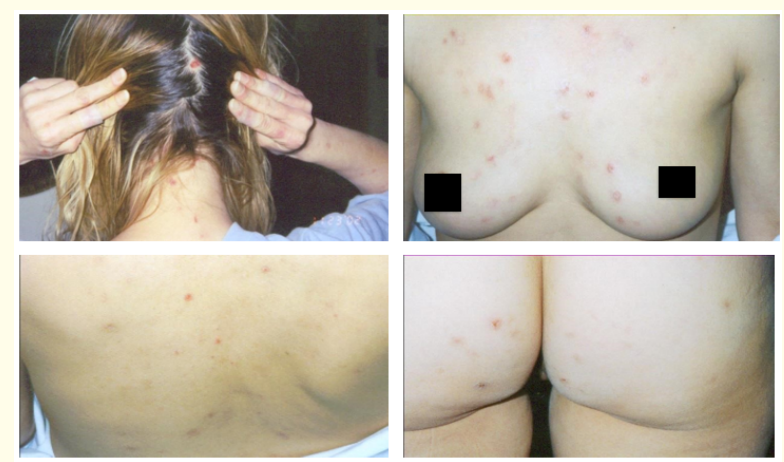

Figure 18

KK: White female from Arizona born on 9-1-1978

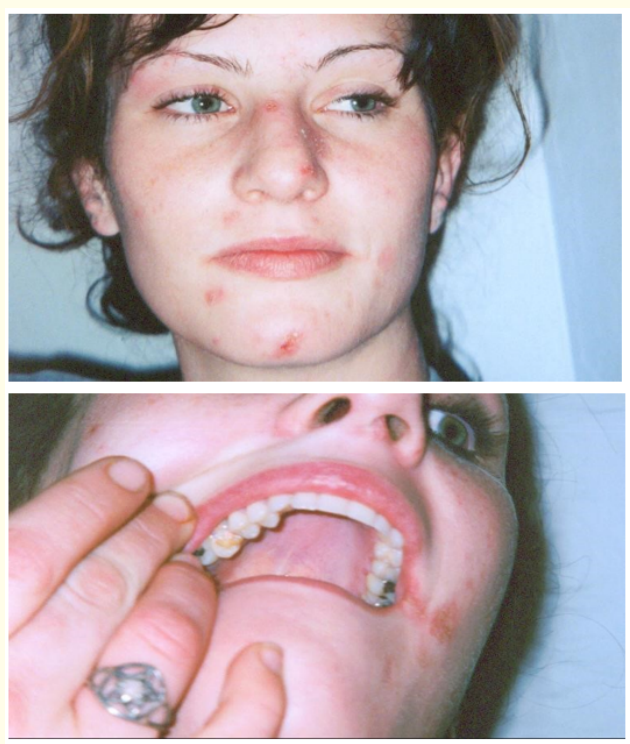

Figure 19
- $\quad 9$ teeth worked on 1992-2002 using SE Bond and Vitre-Bond (dimethacrylate).

- $\quad$ First symptoms in early, 2004:

- Dermatology: Open lesions, itchy and painful sores (also on scalp), elevated tracks and veins, skin infections, peeling, fibers.

- Neurology: Pin prick, crawling and burning sensations, loss of memory, brain fog, poor concentration, vision problems.

- $\quad$ Other: Breathing, bowel, liver and kidney disturbances, swelling, tight chest, vomiting. Inflamed gum, mucoid secretions, dental decay, thrush, nausea, insomnia, fatigue, compromised immunity, trauma.

- $\quad$ Allergic to light and metals.

KK (cont.): Diagnosed with "encrusted psoriasiform dermatitis" on 6-3-04. Recurrent eruptions treated as scabies but recurs. No scabies demonstrated. But breast implants documented. Diagnosed with NCS at PCI on 7-2-2004. Clinical picture compounded by use of methamphetamine (crystal meth).
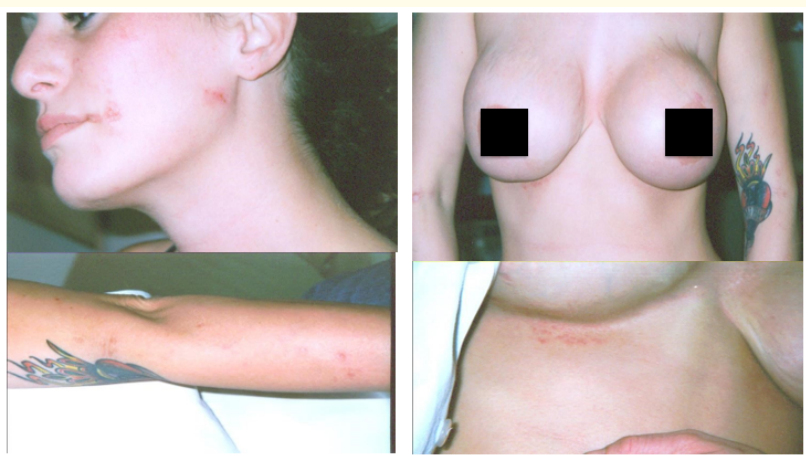

Figure 20

N(A)H: White female from Arizona born 3-8-1948

Not all NCS patients experience marked dermatological symptoms

- $\quad$ Silver fillings replaced with nonmetalic material in 1987. 9 new fillings in 2001-02 using Z250 (dimethacrylate) and Solo plus.

- $\quad$ Skin symptoms occasional and mild (lesions and sores) on head. 
- $\quad$ Neurology: Strong itching, pin prick, crawling sensations (believed to be from mites that were never recovered) brain fog, vision, memory and systemic problems.

- $\quad$ Allergy to metals, penicillin, aspirin, electromagnetic fields and mold, and insomnia.

- Undergoing dental rehabilitation on and off.

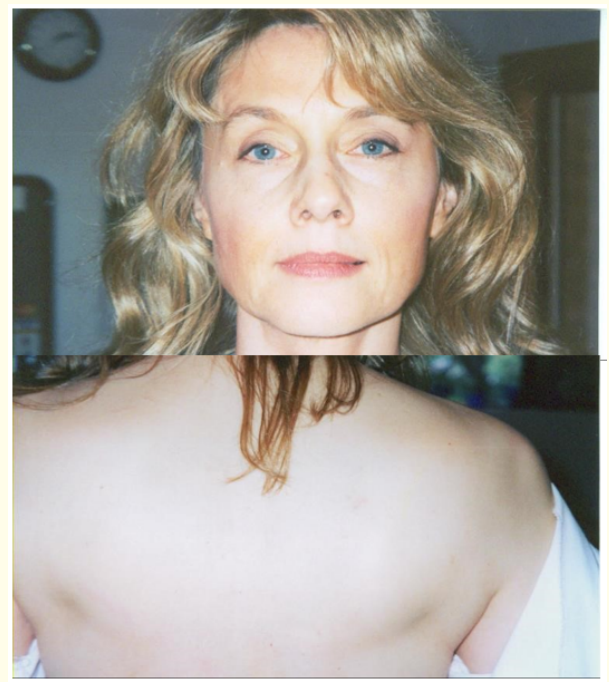

Figure 21

ME: White male from Las Vegas born 8-11-1950 A storage organ case

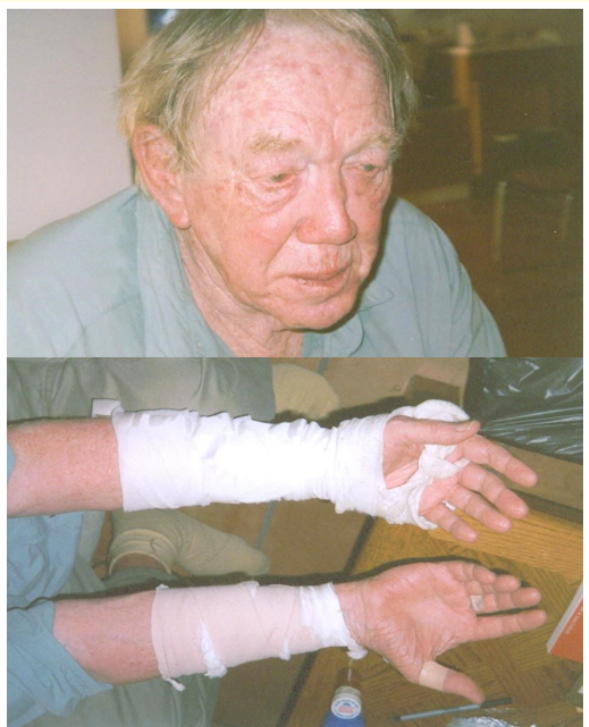

Figure 22
- $\quad$ ME had classical neurological and dermatological symptoms of NCS.

- He lost the last of his original teeth 3 years ago. Dental materials used: unicem, clear fill prime and bond, fugi and rely $\mathrm{x}$ (all methacrylate- and dimethacrylate-based) and Temp bond (zinc oxide) circulating with lymph to storage organs (liver, pancreas, spleen) and continue to cause NCS symptoms after teeth removal.

- He came to see us at PCI on 9-6.

- He promptly followed our protocol and considerable improvement was noted over the next few weeks.

- He was hospitalized for a heart condition and died in the hospital after an injection of narcan on 9-28.

Procedures for rehabilitation

- $\quad$ Personal interview with patient.

- Examination of patient and verifying symptoms: history, photos.

- Examination of dental and related records.

- $\quad$ Patient filling up a questionnaire and documenting symptoms.

- $\quad$ Swabbing patient for bacteriological and fungal infections.

- $\quad$ Establishing a diagnosis and providing report to patient.

- Examination of dental issues.

- Vitamin supplementation.

- Initiate skin and internal cleansing.

- $\quad$ Follow up on progress.

Considerations for the proper selection and use of replacement dental materials

- $\quad$ Examine Material Safety Data Sheets (MSDS) for products used.

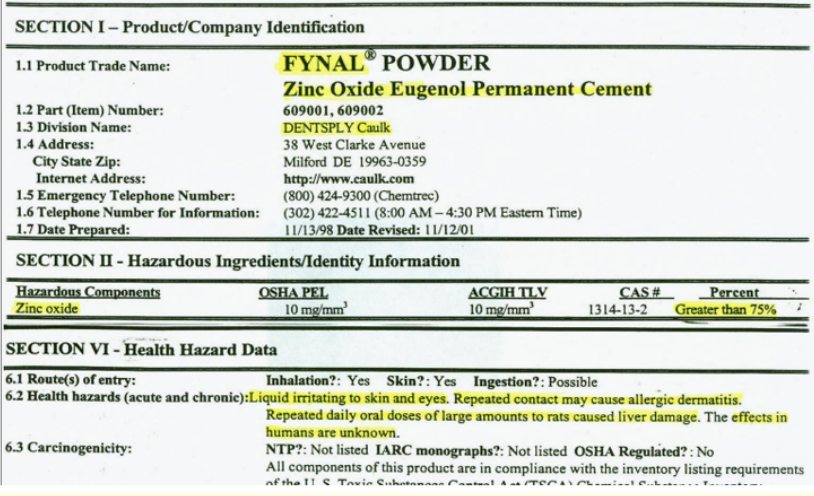

Figure 23 


\section{Considerations}

In selecting dental materials (sealants, bonds, adhesives, etc.) to replace removed materials: consider the following example: Dycal includes zinc oxide, among other ingredients. It is recommended NOT to replace using material that includes zinc oxide such as Durelon even if the compatibility test shows that the patient is not (highly) reactive to Durelon.

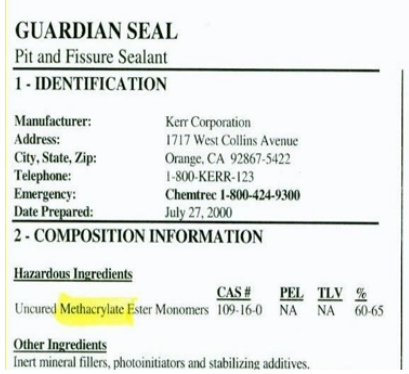

MATERIAL SAFETY DATA SHEET

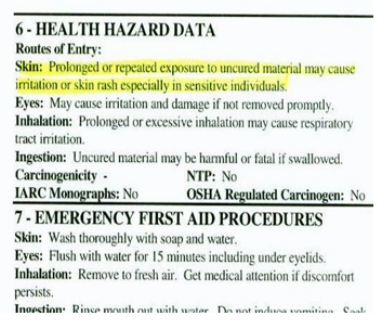

Figure 24

Examples of dental materials containing: methacrylates, dimethacrylates, or 2-hydroxyethyl-methacrylates.

\begin{tabular}{|l|l|}
\hline Adper (3M single bond) & $\begin{array}{l}\text { Gluma Comfort Bond } \\
\text { All-Bond } 2\end{array}$ \\
Bisfil core & $\begin{array}{l}\text { Guardian Seal } \\
\text { Heliomolar }\end{array}$ \\
Bisfil II base paste & Hi-X Base \\
Clearfil SE (bond, primer) & Optibond Solo \\
Cavalite & Solo Plus Bond \\
Filtek & ProBond Adhesive \\
Filtek Z100 Filtek Z250 & Scotchbond Multi-Purpose \\
Fuji 2 & Structure 2 SC \\
Fuji & Vitre-Bond \\
\hline
\end{tabular}

Table 1

Dental material containing : Calcium hydroxide, ethyltoleune sulfonamides, zinc oxide, titanium dioxide and barium sulfate.

\section{Dycal \\ Calcium Hydroxide Composition in Pressure Pack - CFC Free}

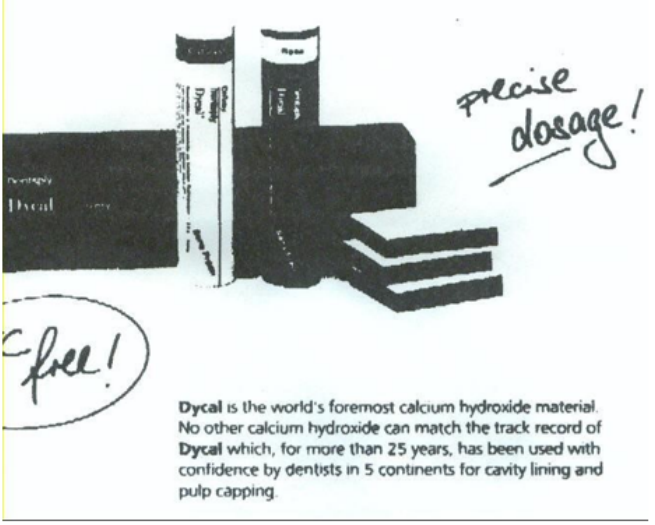

Figure 25

$\begin{array}{ll}\text { - } & \text { Dycal } \\ \text { - } & \text { Life } \\ \text { - } & \text { Sealapex }\end{array}$

Examples of dental materials containing

Zinc oxide

- $\quad$ Cavitec Base

- Cavit

- $\quad$ CRCS (with calcium hydroxide)

- Durelon

- $\quad$ Fi-cem Paste Alecks Zinc (Mizzy)

- Fynal Powder

- Gutta Percha

- IRM powder

- Modern Tenacin

- Pulpdent

- $\quad$ Pulp Canal Sealer powder

- Temp Bond Base

- $\quad$ Tubli-Seal) (with eugenol)

- $\quad 3 R M$ Liquid (with eugenol)

- Sultan (eugenol)

- $\quad$ Hypocal SN (calcium hydroxide) 
On line help

NCS patients who are not able to come to Scottsdale can now access an on line self-help and self-paced program for assessment and treatment by clicking on Morgellons (NCS) on our web site www.parasitetesting.com/morgellons.cfm (Figure 26) and going through the program thus avoiding the costs of travel and accommodations. This is especially helpful for overseas patients.

Morgellons or Neuro-cutaneous Syndrome (NCS)

MAIN MENU
$\Rightarrow$ Home
$\Rightarrow$ About PCI
$\Rightarrow$ Tests
$\Rightarrow$ Products
$\Rightarrow$ Parasite Cleanse
$\Rightarrow$ Parasite Wiki
$\Rightarrow$ Services
$\Rightarrow$ Testimonials
$\Rightarrow$ Morgellons (NCS)
$\Rightarrow$ Consultation
$\Rightarrow$ Publications
$\Rightarrow$ Forms
$\Rightarrow$ Parasites
$\Rightarrow$ Videos
$\Rightarrow$ FAQs
$\Rightarrow$ Login

Figure 26

\section{Introduction}

The terms Morgellons, and Neuro-cutaneous Syndrome (NCS) as characterized by Amin (2001 - 2009) are used interchangeably, yet cautiously, as their symptoms are very similar. While Morgellons has never been researched, the etiological agent (s) and remedies have never been identified, these factors for NCS have been well researched and published in refereed medical journals (see below) and patients have been successfully helped. Only a few highly speculative accounts of a wide assortment of purported infectious agents for Morgellons serve as an awareness call for those suffering comparable symptoms but have not managed to find out where to go for help.
A more recent article on 644 toxic dental products is now available on our web site www.parasitetesting.com and through the Explore web site, vol. 21: 14-23.

A comprehensive study of dental materials and their toxic ingredients associated with Neuro-Cutaneous Syndrome (NCS) and morgellons, with notes on research background from the last page "of dental products..."

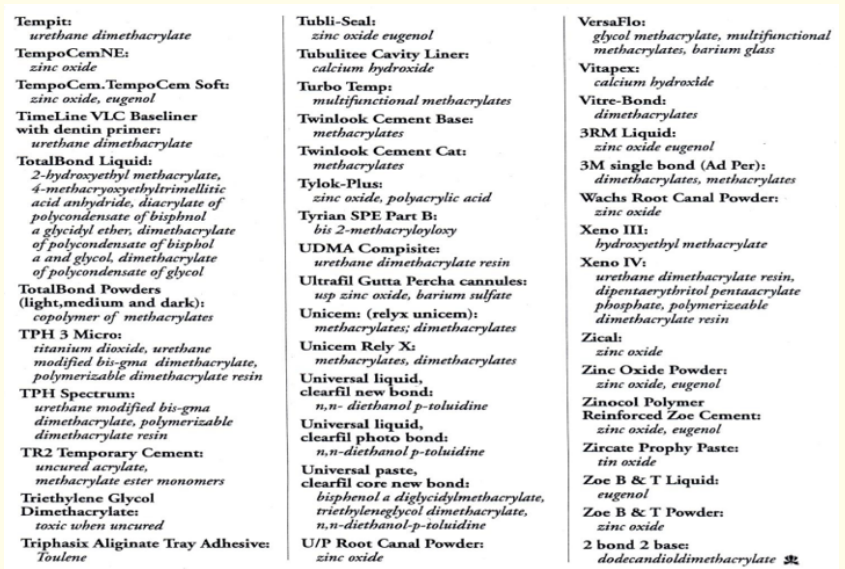

Figure 27

\begin{abstract}
An Overview of Neuro-Cutaneous Syndrome (NCS) with a Special Reference to Symptomology

O By Omar M. Amin, B.SC.. M.SC., Ph.D., USA

Abstract

$A$ detailed analysis of the clinical history of a random sample of 50 NCS patients (9 males, 41 categories, neurological, dermatological (including opportunistic skin infections), systemic, oral, allergic and general. The most common symptoms in each of these categories in the same order are pin prick and crawling sensations, skin the same order are pin prick and crawling sensations, skin lesions and sores, respiratory and bowel disturbances, gum disease, sensitivities to light, noise and mold, and fatigue and insomnia. Symptoms were relatively similar in both sexes. These results are tabulated and their biological foundation explained. The misdiagnosis of NCS by medical professionals is discussed. NeS symptoms in toothless patzents and those on recreational drugs are described. Over 360 dental toxins are placed in four major categories and their mode of action explained. Incubation perzod varied between a few hours to 28 years. Our protocol for rehabilitation is included. All patients following and completing our rehabilitation protocol recovered.
\end{abstract}

Figure 28 


\begin{tabular}{|c|c|c|c|c|c|c|}
\hline & & Neurological Syr & $\begin{array}{l}\text { able \#1 } \\
\text { ptoms in } 50\end{array}$ & & & \\
\hline & & & Putients with & toms $1 \%$ & & \\
\hline & & & & Yof symity & Eind soi pou & \\
\hline & Males $(n=9)$ & Females $(n=41)$ & Totol $(n=50)$ & Severe & Moderate & light \\
\hline Skin irritation & $9(100)$ & $33(80)$ & $42(84)$ & $35(83)$ & $7(17)$ & 0 \\
\hline Pin prick sensations & $9(100)$ & 38 (92) & $47(94)$ & $34(72)$ & 12(26) & $1(2)$ \\
\hline Growling sensations & $8(88)$ & $38(93)$ & $46(92)$ & $32(69)$ & $13(28)$ & $1(2)$ \\
\hline Burning sensations & $8(88)$ & $28(68)$ & $36(72)$ & $21(58)$ & $12(33)$ & $3(8)$ \\
\hline Movement sensations & $9(100)$ & $39(95)$ & $48(96)$ & $27(56)$ & $15(31)$ & $6(13)$ \\
\hline Loss of memory & $8(88)$ & $35(85)$ & $43(86)$ & $21(49)$ & 18 (42) & $4(9)$ \\
\hline Broin fog & $8(88)$ & $33(80)$ & $41(82)$ & $26(63)$ & $13(32)$ & $2(5)$ \\
\hline Poor concentration & $9(100)$ & 39 (95) & 48(96) & $29(60)$ & $14(29)$ & $5(10)$ \\
\hline Body tremors & $7(77)$ & $22(54)$ & $29(58)$ & $9(31)$ & $10(34)$ & $10(34)$ \\
\hline Vision problems & $7(77)$ & $33(80)$ & $40(80)$ & $17(43)$ & $18(45)$ & $5(13)$ \\
\hline
\end{tabular}

Figure 29

\section{The Face of Neuro-cutaneous Syndrome (NCS): New Cases, Recovery, and Perspectives}
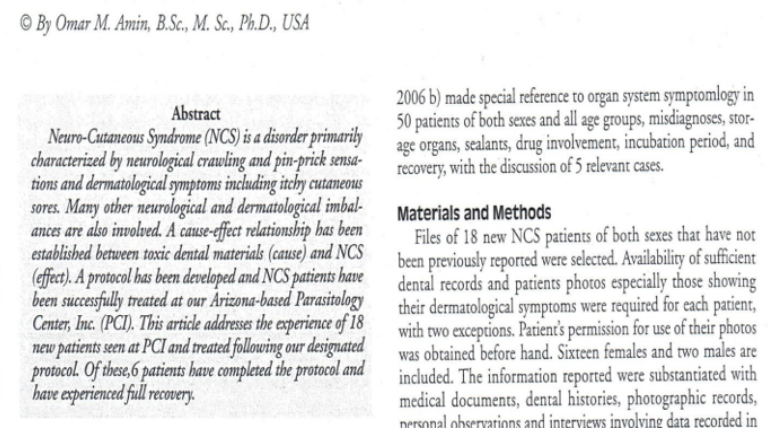

Figure 30

\section{Recovering from Morgellons and Neuro-cutaneous Syndrome (NCS): Patients' Perspectives}

( $)$ By Omar M. Amin, B.Sc, M. Sc, Ph.D., USA

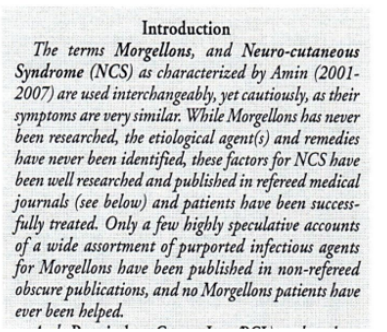

Most people have had dental work. Many have various degrees of sensitivity to some dental materials to which their bodies manifest varied intensities of symptoms. This epidemic-in-disguise has been routinely misdiagnosed by medical professionals who often label patients as delusional because of their unfortunate description of their neurological symptoms (actually catsed by nerve damage as having been caused by parasite infections. Amin (2004 pecifically addressed this issue while discussing the clinical history of 24 NCS patients. Of these patients, 7 who have followed our protocol and completed treatment, by the time of publication, have experienced full recovery. Amin (2005) provided an annotated list of about 40 dental materials that have been involved in the causation

Figure 31
Recent original publications

- Journal of Parasitology. Photon 104 (2014) 139-144.

- Original Research Article. ISJN: 3729-2384.

The misdiagnosis of "delusional parasitosis" Omar M. Amin*

\section{Abstract}

The body of knowledge on delusional parasites incriminates "delusional patients" as having imaginary external and internal symptoms that feel like parasite movements, dismisses them as psychiatric cases, and confuses cause with effect. We are critical of these arguments and believe that the symptoms of crawling and pin-pricking are genuine; just misinterpreted as parasite movements. Based on our research on over 1000 cases.

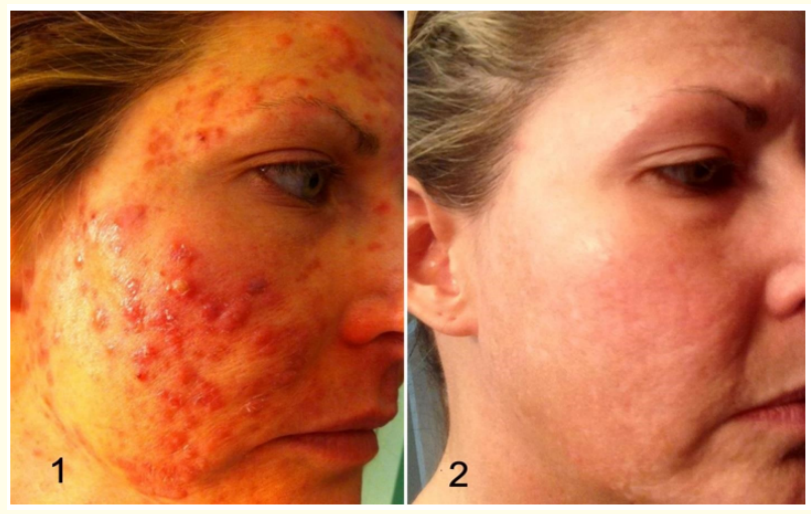

Figure 32

Above Figure indicates SH before and after recovery. (1) The face of SH showing the dermatological symptoms of Neurocutaneous Syndrome at the height of intensity before coming to PCI for diagnosis and treatment. (2) SH's face after her symptoms have totally resolved following dental rehabilitation and detoxification as per the PCI protocol Sep. 14, Vol. 8, No. 9 Journal of Life Sciences, !SSN 1934-7391, USA.

The Case Against Delusional Parasitosis Omar M. Amin Parasitology Center Inc. (PCI), 11445 E.Via Linda \# 2-419, Scottsdale, 85259, Arizona.

Received: August 29, 2014/Accepted: September 30, 2014/Published: September 30, 2014.

GC first came to see us at PCI in May, 2010 with full blown classical neurological and dermatological NCS symptoms (Figure). She 
was clearly NOT a case of delusionary parasitosis. Her more recent dental work included restoration of tooth no. 14 (a 30-year old root canal) on December 11, 2006. Surgical and non-surgical endodontic treatments of tooth No. 14 were completed on the same date. This consisted of Retreatment and apicoectomy with MTA Retrofill procedures. On December 22, 2006 she had a crown porcelain-fuse high noble and a build up with pins on the same tooth. On January 25, 2007 she had an amalgam filling in tooth no. 2 . These dental events immediately preceded the onset of her recent symptoms so graphically described in her anguished personal narrative.

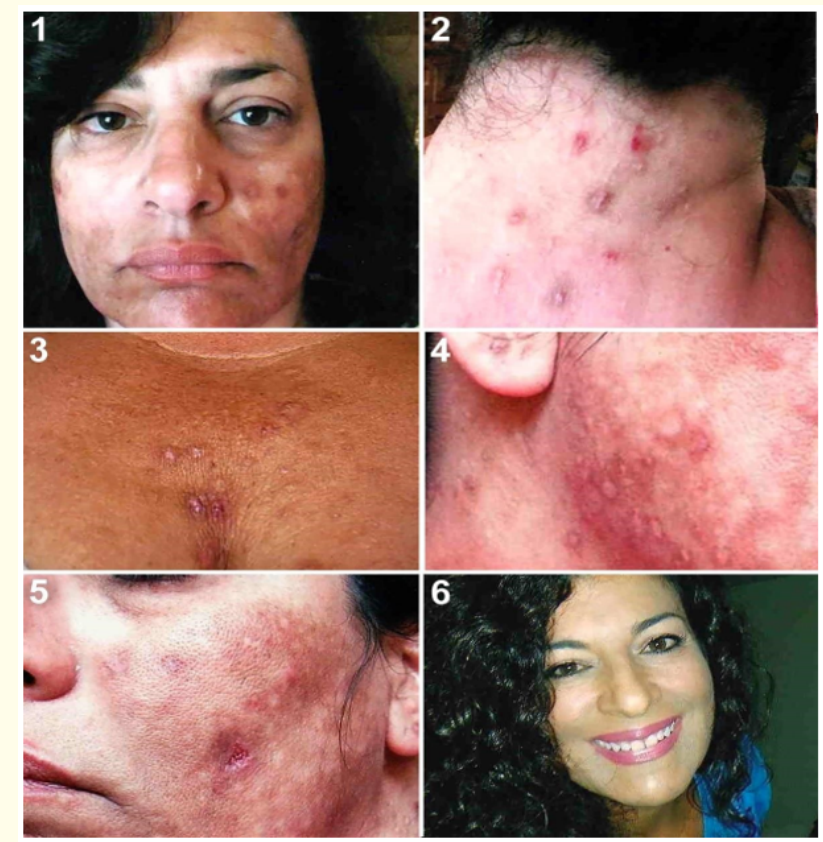

Figure 33

MS is a white female from New Jersey born in 1955. She was diagnosed at PCI in July, 2006 with "classical symptoms of NCS, especially dermatological".

Dermatological symptoms were of constant nature and started in April, 2006.

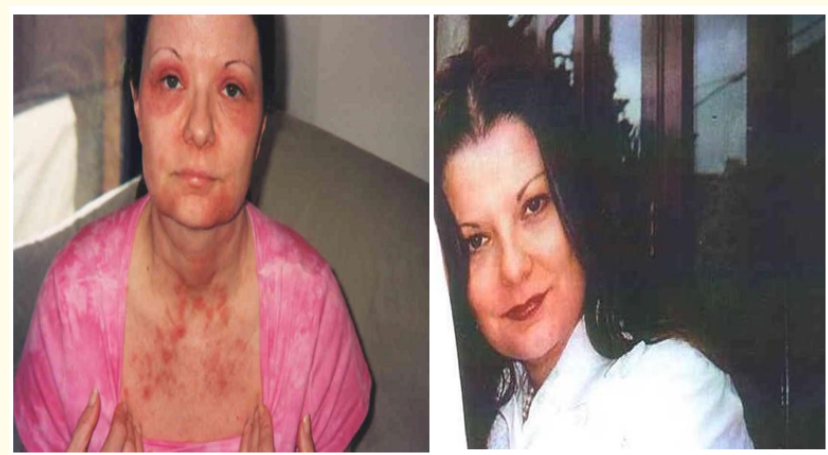

Figure 34

MS (Cout.)

The case of MS represents one more case in which the relationship between the dental materials used (cause) and the manifestation of NCS symptoms (effect) is well documented. MS experienced full recovery a few months later. No recurring symptoms have been reported for the last nine years since the onset of the above episodes in 2006 and subsequent recovery.

JK is a white woman from Wisconsin. She was 54 years old when she came to see us at PCI in March, 2004 when the picture (Figure), among others, was taken. She had all the classical dermatological and neurological symptoms of NCS. Her dental history indicated the use of Gutta Percha and Sultan for four root canal procedures between 1996 and 1998.

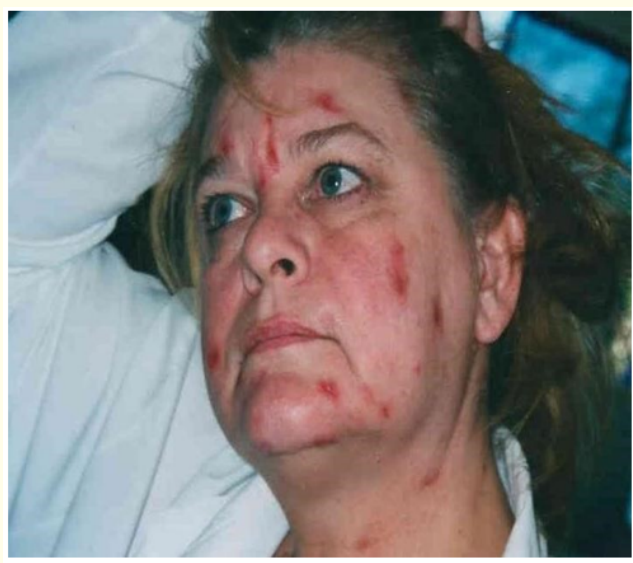

Figure 35 
She presented with sores and lesions allover her body. The sores started early in the mid 1990's but suspected skin "parasites" in the summer of 2003. She has tried Ivermectin, among other skin parasite treatments but at no avail. It was not a parasitic issue. She was given our protocol. A culture of her skin swabs demonstrated a secondary infection with Staphylococcus aureus coagulose positive. In April, 2005, she wrote that she had gotten much better.

MM is a white woman from Arizona. Born in 1941, she was 54 years old when she came to see us at PCI in September of 2003 when this picture (Figure) was taken. At that time, the sore on her forehead was 26 years old. Her first dental work was when she was 11 when living in the Virgin Islands.

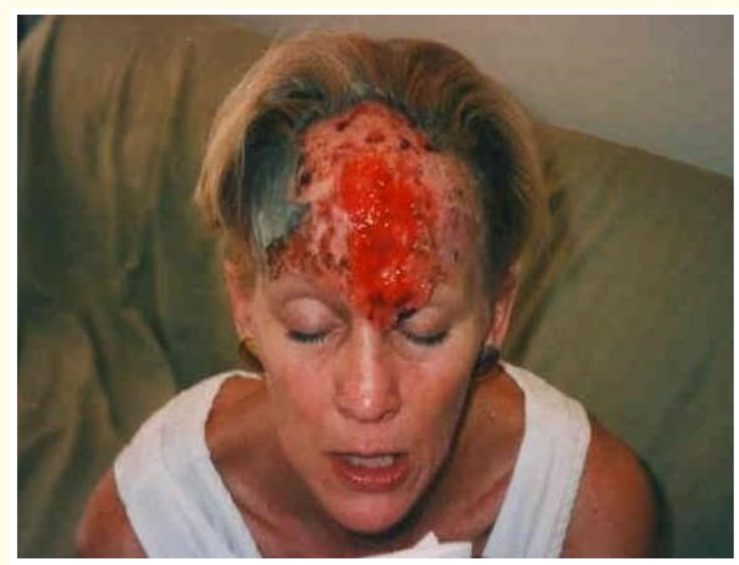

Figure 36

The most prominent was a coalesced large oozing sore on her forehead which started in 1977 when she lived in Florida and Maine. She started feeling biting sensations at that time.

Initially, activity (running in a cycle of 17 - 27 days) started with the skin becoming shiny and red in perfect circle. This is followed by biting sensations where small bumps appeared. The sensations turned into stinging and burning, like acid, then the sore occasionally crusted.

\section{Conclusions}

- The literature on delusional parasitosis dismisses "delusional patients" as psychiatric cases with imaginary external and internal symptoms that feel like parasite movements.
- We have researched over 1000 "delusional" patients (Morgellons cases included) since 1996 at PCI. We have concluded that the internal sensations of crawling and pin-pricking, often confused with parasite movement, are actually caused by toxicity from exposure to toxic chemicals including but not limited to dental materials that interfere with the propagation of normal nerve impulse.

- We also have evidence that external parasites/organisms including springtails (Collembola), other arthropods, bacteria, and fungi represent only opportunistic infections of skin sites compromised by toxins. The elimination of toxins from the skin will cause breaks that will allow the establishment of external infections.

- We have described a new pathological disorder that we called Neurocutaneous Syndrome because of the neurological and the dermatological symptoms that characterize it. We have established a cause and effect relationship. Patients following our protocol invariably recover.

\section{Bibliography}

1. Amin OM. "Facial cutaneous dermatitis associated with arthropod presence”. Explore 7 (1996): 62-64.

2. Amin OM. "Neuro-cutaneous Syndrome (NCS): a new disorder". Explore 10 (2001): 55-56.

3. Amin OM. "On the diagnosis and management of Neuro-cutaneous Syndrome (NCS), a toxicity disorder from dental sealants". Explore 12 (2003): 21-25.

4. Amin OM. "Dental sealant toxicity: Neurocutaneous Syndrome (NCS), adermatological and neurological disorder". Holistic dentistry Association Journal (2004): 1-15.

5. Amin OM. "On the diagnosis and management of Neuro-Cutaneous Syndrome, a toxicity disorder from dental sealants". Journal of the California Dental Association 32.9 (2004): 657663.

6. Amin OM. "On the diagnosis and management of Neuro-cutaneous Syndrome (NCS), a toxicity disorder from dental sealants". Townsend Letter 276 (2006): 85-90.

7. Amin OM. "On the course of Neurocutaneous Syndrome (NCS) and itsPseudo-diagnosis by medical professionals". Explore 13 (2004): 4-9. 
8. Amin OM. "Dental products causing Neuro-cutaneous Syndrome (NCS) symptoms in NCS patients". Explore 14 (2005): 57-64.

9. Amin OM. "An Overview of Neuro-cutaneous Syndrome (NCS) with a special reference to symptomology". Explore 15 (2006): 41-49.

10. Amin OM. "The face of Neuro-cutaneous Syndrome (NCS): new cases, recovery, and perspectives". Explore 16 (2007): 5464.

11. Amin OM. "Recovery from Morgellons and Neuro-Cutaneous Syndrome (NCS): patients perspectives". Explore 18 (2009): 25-33.

12. Amin OM. "In their own words OR: symptoms of Morgellons and Neurocutaneous Syndrome (NCS) upon first examination at the Parasitology Center, Inc. (PCI)". Explore 19 (2010): 2431.

13. Amin OM. "A comprehensive study of dental materials and their toxic ingredients associated with Neuro-cutaneous Syndrome (NCS) and Morgellons, with notes on research background". Explore 21 (2012): 14-23.

14. Amin OM. "The misdiagnosis of "delusional parasitosis". Journal of Parasitology 104 (2014): 139-144.

15. Amin OM. "The case against delusional parasitosis". Journal of Life Sciences 8.9 (2014): 731-739.

16. Amin OM. "Disseminated dermatological symptoms in chronic cases of Neurocutaneous Syndrome (NCS) or Morgellons". Clinical Microbiology Case Reports 1.2 (2015).

\section{Volume 2 Issue 11 November 2019}

(C) All rights are reserved by Omar M Amin. 